\title{
LISA PATHFINDER OPTICAL INTERFEROMETRY
}

\author{
C Braxmaier ${ }^{a}$, G Heinzel ${ }^{b}, \mathrm{~K} \mathrm{Middleton}^{d}, \mathrm{M} \mathrm{Caldwell}^{d}$, $\mathrm{W} \mathrm{Konrad}^{a}$, $\mathrm{H} \mathrm{Stockburger}^{a}$,

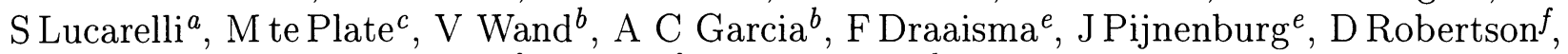 \\ C Killow ${ }^{f}, \mathrm{H} \mathrm{Ward}^{f}, \mathrm{~K} \mathrm{Danzmann}^{b}$ and U Johann ${ }^{a}$ \\ ${ }^{a}$ EADS-Astrium GmbH, 88039 Friedrichshafen, Germany; \\ ${ }^{b}$ Max-Planck-Institut für Gravitationsphysik (Albert-Einstein-Institut), Callinstrasse 38, \\ D-30167 Hannover, Germany; \\ ${ }^{c}$ ESA-ESTEC, NL-22000 AG Noordwijk, The Netherlands; \\ ${ }^{d}$ Rutherford Appleton Laboratories, Chilton, Didcot Oxfordshire OX11 0QX UK; \\ ${ }^{e}$ TNO TPD Institute of Applied Physics P.O. Box 155, 2600 AD Delft, The Netherlands; \\ $f_{\text {Glasgow University, Glasgow G12 8QQ, UK }}$
}

\begin{abstract}
The LISA Technology Package (LTP) aboard of LISA pathfinder mission is dedicated to demonstrate and verify key technologies for LISA, in particular drag free control, ultra-precise laser interferometry and gravitational sensors. Two inertial sensors, the optical interferometry in between combined with the dimensional stable Glass ceramic Zerodur structure are setting up the LTP. The validation of drag free operation of the spacecraft is planned by measuring laser interferometrically the relative displacement and tilt between two test masses (and the optical bench) with a noise levels of $10 \mathrm{pm} / \sqrt{\mathrm{Hz}}$ and $10 \mathrm{nrad} / \sqrt{\mathrm{Hz}}$ between $3 \mathrm{mHz}$ and $30 \mathrm{mHz}$. This performance and additionally overall environmental tests was currently verified on EM level. The OB structure is able to support two inertial sensors $\left(\approx 17 \mathrm{~kg}\right.$ each) and to withstand $25 \mathrm{~g}$ design loads as well as $0 \cdots 40^{\circ} \mathrm{C}$ temperature range. Optical functionality was verified successfully after environmental tests. The engineering model development and manufacturing of the optical bench and interferometry hardware and their verification tests will be presented.
\end{abstract}

Keywords: LISA pathfinder, optical interferometry, optical bench, gravitational wave, inertial sensor, Zerodur structure

\section{INTRODUCTION}

The LISA Pathfinder spacecraft will contain a European LISA Technology Package (LTP) and a similar USsupplied package (ST-7). Both packages consist of two free-floating test masses, each in capacitive sensor cages ("Gravitational Reference Sensors"), that are the heart of the drag-free control and an essential part of the LISA mission. ${ }^{1}$ The European package LTP and the US package ST-7 ${ }^{2}$ will have coordinated differences in construction such as to maximize the scientific returns of the mission. The purpose of both packages is to test a variety of operational modes of the Gravitational Reference Sensors together with their associated $\mu \mathrm{N}$-thrusters and drag-free loops, and to verify their performance and noise behaviour. Both packages will be launched together in 2007 on an ESA satellite, the LISA Pathfinder mission (formerly called SMART-2).

The maximum allowed acceleration noise for the LTP test masses is one order of maximum less demanding compared to LISA: $3 \times 10^{-14}\left(1+(f / 3 \mathrm{mHz})^{2}\right) \mathrm{m} / \mathrm{s}^{2} \sqrt{\mathrm{Hz}}$ within $f=1 \mathrm{mHz} \cdots 30 \mathrm{mHz}$. The residual acceleration budget for the LTP test masses converted to maximum displacement noise with margins to be provided by the laser interferometers is shown in fig. 1 (left).

Within an ESA study EADS Astrium GmbH developed an engineering model of the LPF optical bench for LTP (EM OB), which is shown in the photograph Fig. 1, right. The EM OB team was composed by EADS Astrium GmbH (prime), Albert-Einstein-Institut (science, preinvestigations, interferometry, optical design, optical tests), Rutherford-Appleton-Laboratory (preinvestigations, detailed optical design, manufacturing), University of

Further author information: (Send correspondence to C Braxmaier)

E-mail: claus.braxmaier@astrium.eads.net, Telephone: ++49 (0)7545 82493 

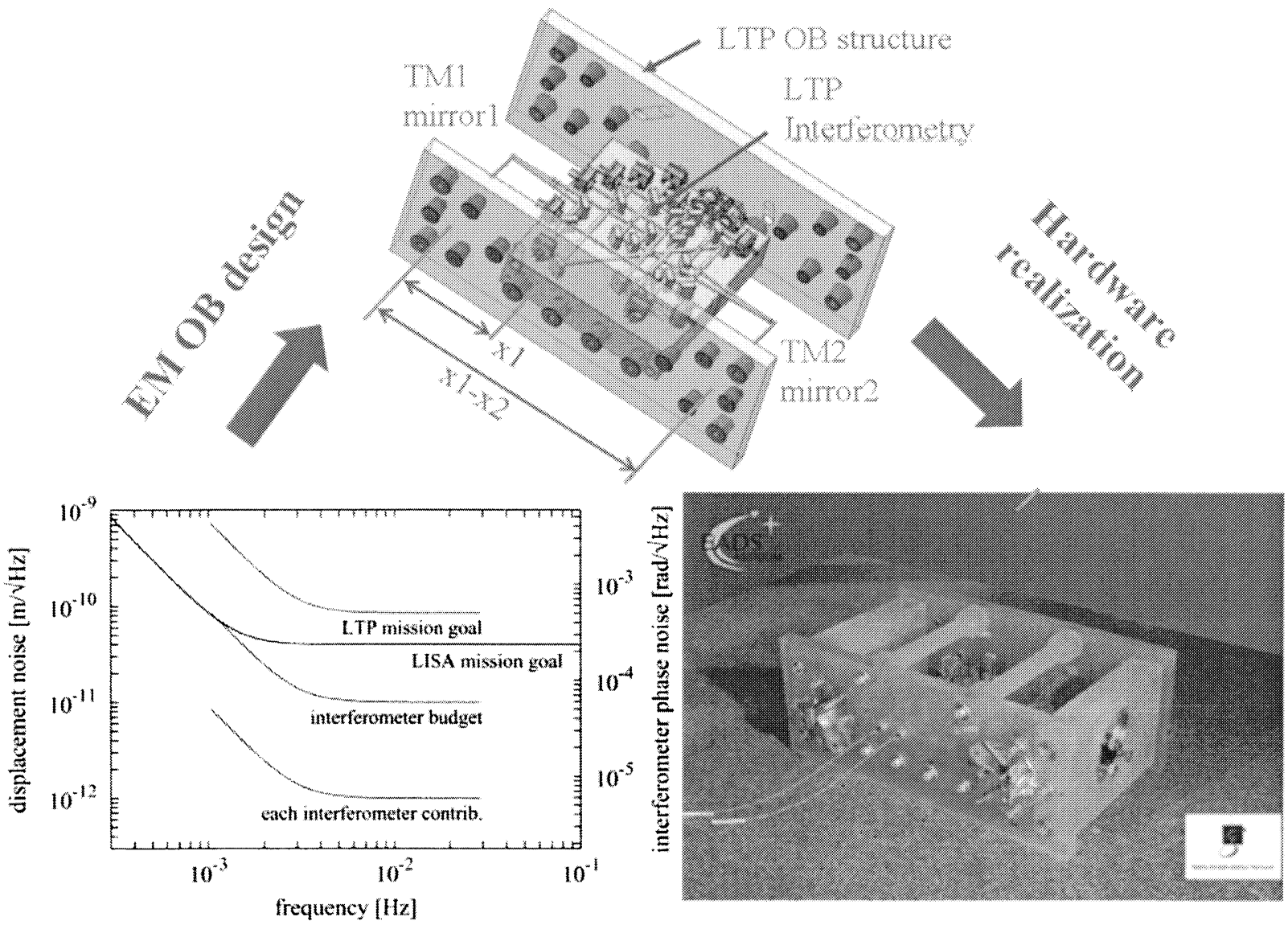

Figure 1. Left: Noise budgets for the LTP, its interferometer and each individual interferometer noise source. The LISA total displacement metrology noise budget is also shown for comparison. Above: Design of EM OB with interferometry and structure we developed. Shown are optics, laser beams, adjusted to reflection points (test mass (TM) positions) and the main measurands $x_{1}-x_{2}$ (relative distance TM1 - TM2) and $x_{1}$ (TM1 to OB). Also visible is the integration technology between the Zerodur Glass parts via inserts. Right: Integrated EM OB hardware fulfilling the metrology requirements - with dummy mirrors representing the test masses (TM) for performance tests and brackets for attachment to the SC.

Glasgow (preinvestigations, consultants hydroxide catalysis bonding), Contraves Space (modulation unit GSE), TNO-TPD (performance and environmental tests). The study was currently successfully finalized. This paper presents the development, manufacturing, assembly, and integration as well as the verified performance and environmental test results of the optical bench hardware on engineering model level.

\section{HARDWARE REQUIREMENTS TO LISA PATHFINDER OB}

Again, the requirements of LISA pathfinder have been relaxed by a factor of ten both in frequency and sensitivity as compared to LISA (see fig. 1). The interferometric sensing must be able to monitor the test mass position along the sensitive axis (called the x-axis) with a displacement noise level of $10 \mathrm{pm} / \mathrm{Hz}^{1 / 2}$ between $3 \mathrm{mHz}$ and $30 \mathrm{mHz}$, relaxing as $1 / \mathrm{f}^{2}$ towards $1 \mathrm{mHz}$. The interferometer must do so without exerting any influence on the test masses that might lead to a motion above that level. The interferometer noise budget for the optical pathlength is chosen a factor of 17 below that LPF mission goal. Each individual noise contribution is allocated another factor of 10 below that (see figure1). This conservative requirement takes into account the possibility that some noise sources are correlated and might add linearly instead of quadratically. The angular metrology requirement is $10 \times 10^{-9}\left(1+(f / 3 \mathrm{mHz})^{2}\right) \mathrm{rad} / \sqrt{\mathrm{Hz}}$ within $f=1 \mathrm{mHz} \cdots 30 \mathrm{mHz}$. 
Launch mode requires an opto-mechanical structure withstanding $25 \mathrm{~g}$ design loads. A temperature range to be survived by the EM OB of $0 \cdots 40^{\circ} \mathrm{C}$ was required. To ensure optimum operation of the LPF metrology the EM OB structure must also provide an ultra-high dimensional stability of maximum $1 \mathrm{pm}$ over approx $0.5 \mathrm{~m}$, which restricted the choice of materials.

\section{LISA PATHFINDER EM OB DESIGN APPROACH}

The path from the main requirement (maximum allowed displacement metrology noise) to design and realization of the LISA pathfinder OB is shown in fig. 1.

Optical interferometry: The interferometer of the LTP as diagnostic tool used to continuously monitor the test masses in all operating modes is measuring the distance between the two test masses (called $x_{1}-x_{2}$ ), the position of one test mass with respect to the optical bench (called $x_{1}$ ), the differential alignment of the two test masses (with two sets of measurements: DC and Differential Wavefront Sensing), and the alignment of one test mass with respect to the optical bench. Its principle of operation and general design are described in References ${ }^{3}$ and, ${ }^{4}$ and is shown in figure 1 . The baseline is a heterodyne Mach-Zehnder interferometry.

The main purpose of the LTP interferometer is to verify the performance of the gravitational sensors by monitoring the distance and relative tilt between two test masses, which are approximately $30 \mathrm{~cm}$ apart. For the interferometers a space of approximately $200 \times 200 \mathrm{~mm}^{2}$, in the form of an optical bench (a slab of Zerodur), centered between the two test masses will be available. Optical components are mounted by hydroxide catalysis bonding* on the LTP interferometer baseplate, which will be in a thermally highly stable environment.

EM OB Design: Main part of the EM OB is the interferometer baseplate between two reinforcement side slabs connected with an insert based technology, which was also environmentally and in performance verified within the study. The photo in figure 1 shows also dummy mirrors attached to Zerodur plates as test equipment for the performance tests. These mirrors acted as reflectors representing the test masses.

For environmental tests we loaded the EM OB with mechanical and thermal representative dummies for the two inertial sensors mentioned above. The dummies masses are representative in moment of inertia, mass, center of gravity and Eigenfrequency - in combination with Titanium as spacer material between the side slabs to be also thermally representative - see figures 7 and 9.

\section{EM OPTICAL BENCH MANUFACTURING}

To fulfill the demanding launch load and operation mode requirements for the payload mentioned above, the whole assembly was reinforced by applying two similar side slabs (see for example figure 1).

The masses of the two inertial sensors $(\approx 17 \mathrm{~kg}$ each $)$ in regard with the launch load requirement mainly drove the design together with the required high dimensional stability between the test masses (operation mode), which restricted tremendously the selection of applicable materials to ultra low expansion materials. A trade off results in the use of Zerodur, mainly driven by delivery time and costs.

Thermo-mechanical properties Glass ceramic materials depict maximum allowable mechanical stress in the order of $10 \mathrm{MPa}$, which is quite small compared to metal material whose ultimate stress could exceed $1 \mathrm{GPa}$.

Zerodur depicts a coefficient of thermal expansion in the order os $2 \times 10^{-8} / \mathrm{K}$ roughly at room temperature, which drives the temperature stability requirement of the optical bench to $1 \times 10^{-4} \mathrm{~K} / \sqrt{\mathrm{Hz}}$ for an allowable single error of $1 \mathrm{pm} / \sqrt{\mathrm{Hz}}$ within the MBW, based on $0.5 \mathrm{~m}$ total length.

\footnotetext{
*This optical bonding method was developed at University of Glasgow and adapted for the LTP optical bench within the development by RAL supported by University of Glasgow.
} 

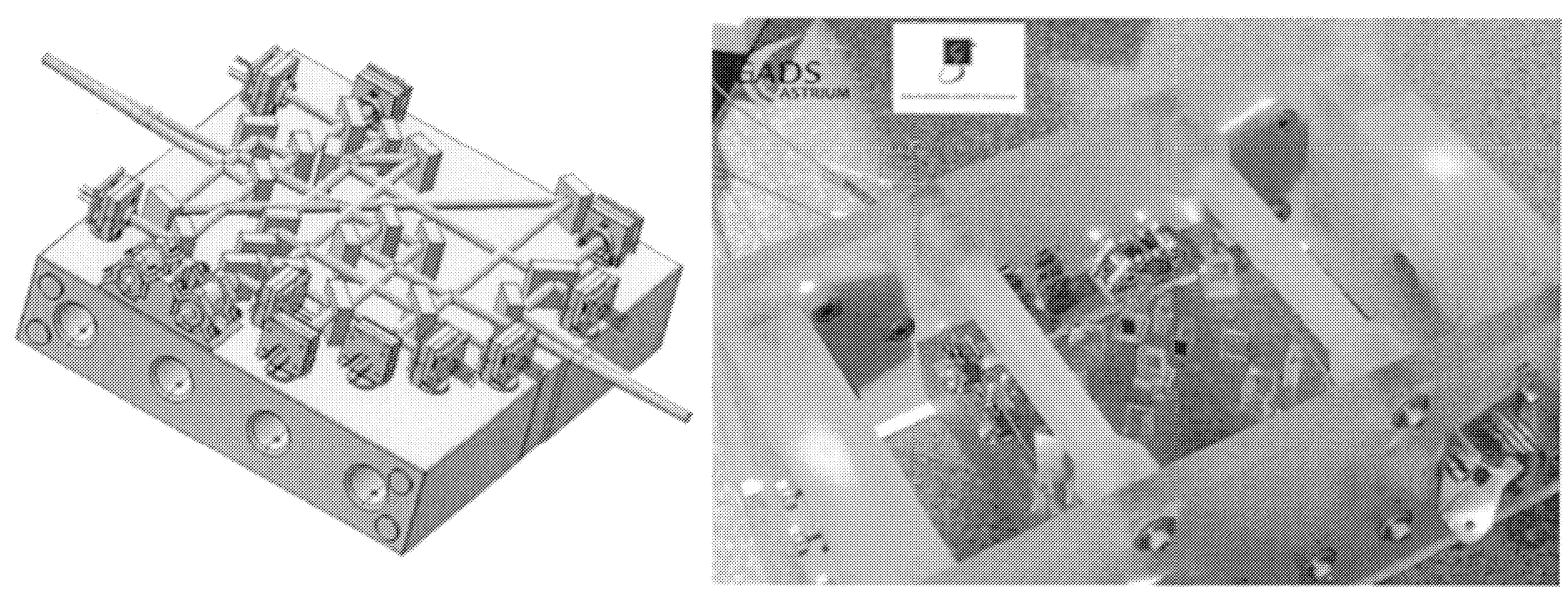

Figure 2. Left: Design of interferometer baseplate with optics and commercial-of-the-shelf fiber launchers. The laser beams are also indicated. Two reflection points can be seen (TM positions). Right: Hardware of the interferometer baseplate integrated with reinforcement side slabs, also made from Zerodur.

Interferometer baseplate and optical components Again, to achieve the required dimensional stability, the interferometer baseplate is fabricated from a single piece of Zerodur, of dimensions $200 \mathrm{~mm} \times 200 \mathrm{~mm} \times 45 \mathrm{~mm}$. The baseplate is polished to surface accuracies of $\lambda / 10$ and angular tolerances of 10 arc seconds in order to provide a sufficiently flat surface for component mounting and to meet interface requirements with other parts of the system. The optical components consist of mirrors and beamsplitters - of dimensions $15 \mathrm{~mm} \times 20 \mathrm{~mm} \times 7 \mathrm{~mm}$ thick - and are made from fused silica: a material chosen to give the lowest possible coupling factor from thermally driven changes in dimension and refractive index. Again, the components must be made to very high accuracy, with angular tolerances of 2 arc seconds, in order to preserve alignment. The design and realization see fig. 2.

The two fibre-coupled input beams are brought onto the optical bench using commercial-of-the-shelf fibre injector assemblies (from Schaeffter \& Kirchhof, Germany), held in a purpose-built titanium mount. The mount is adjustable, by means of shims, to enable the input beams to be aligned, both with respect to each other, and also with respect to the baseplate. Using this technique, beam alignments on the order of $100 \mu$ rad have been achieved. The dimensional stability of the input optics is somewhat less critical than the optics on the interferometer baseplate, and thus the whole mount can be bonded to the baseplate using an epoxy adhesive.

The output photodiodes are housed in purpose-built assemblies, which provide adjustment, to allow the photodiode to be centered on the beam and then locked in position. The housing incorporates a small called pcb (company name) and miniaturized connector for signal read-out. The assemblies are bonded directly to the baseplate, using an epoxy adhesive.

For the DWS method quadrant diodes (from AME, Norway) were implemented ( 16 pieces). To monitor the laser radiation intensity noise two single element photodiodes diodes, one for each metrology laser beam, are used (see section in figure 3 and 2).

To ensure sufficient stability, all optical component mounting within the interferometer must be achieved using only glass materials - a severe constraint on the opto-mechanical design. Conventional joining methods, such as optical cements cannot be used to bond the components to the baseplate, because they do not provide sufficient thermal stability and strongly related to that dimensional stability . Instead, hydroxyl-catalysis bonding, a technique which results in an extremely thermally stable and strong bond layer, is used to join the pieces to the baseplate.

The majority of optical components are placed onto the baseplate without any fine optical alignment, by means of a precision machined template. The placement accuracy of each component using this method is comparatively low - of the order of $\pm 100 \mu \mathrm{m}$ - but any accumulated misalignment can be compensated for at the 
final output beamcombiner. The final beamcombiner in each interferometer path is adjustable in two degrees of freedom (translation and rotation) which enables the interfering beams to be co-aligned for both angle and position. Purpose built manipulators, driven by micrometer stages, and designed to work within the space constraints imposed by the optical layout, are used to position the beamcombiners before final bonding. Using this technique, beam co-alignment of better than $100 \mu \mathrm{rad}$ and $100 \mu \mathrm{m}$ has been achieved. Fringe contrast in excess of $80 \%$ has been obtained from all channels of the completed interferometer. The interferometer baseplate assembled with optics can be seen in a section of figure 3 and 2 .

Reinforcement slabs Both slabs are also made form Zerodur to guarantee the dimensional stability between the two inertial sensors. Critical is the interface between the OB Zerodur and the IS Titanium metal housing, escpecially wrt differential CTE effects. This was solved via partially flexible interface flanges made from Titanium to the Glas ceramic slabs using the EADS Astrium GmbH insert technology. Partially flexible means the flange may compensates differential CTE effetcs in normal direction to the baseplate, perpendicular to the sensitive axis $x$ to reduce stress to the Zerodur.

\section{METROLOGY PERFORMANCE VERIFICATION}

Figure 3 shows an picture of the actual interferometer baseplate, the whole optical bench with the photodiode harness and NTC sensors attached and dummy mirrors (due to lacking real test masses).

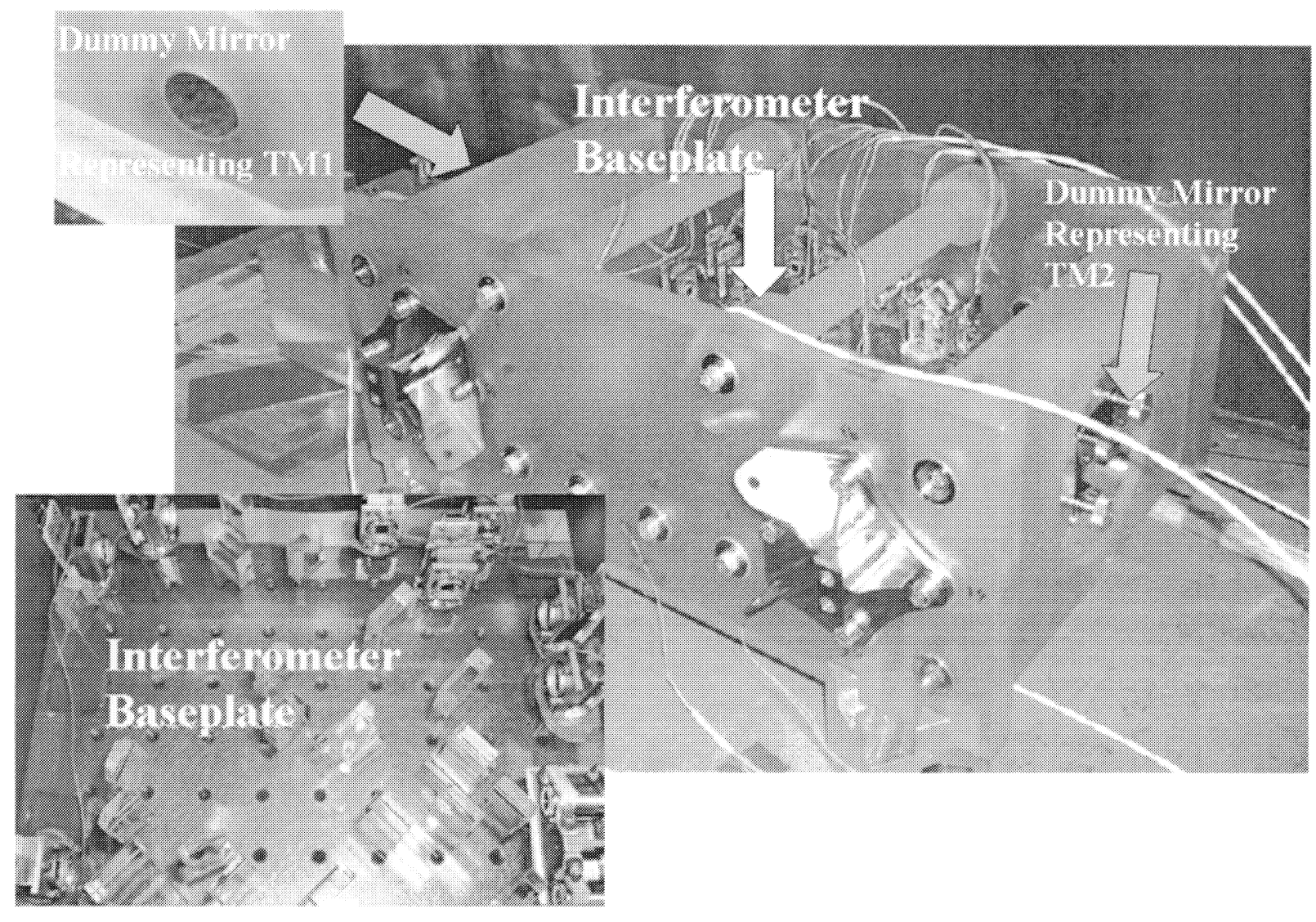

Figure 3. Picture of the EM OB with dummy mirrors and interferometer baseplate. Also shown: photodiode harness and NTC sensors attached to the EM OB. 
They were made from Zerodur with a gold coating and mounted in a commercial precision mirror mount. The mount was fixed in a Zerodur adapter plate connected to the H-structure. The metal components of the mirror mount had a much larger thermal expansion than the optical bench, such that the interferometric length measurements $x_{1}$ and $x_{1}-x_{2}$ at low frequencies were dominated by these thermal expansions. The mirror mounts could easily be adjusted by hand, using the interferometer signals as displayed on the phasemeter. After some exercise, one end mirror could be aligned from scratch within a few minutes.

The purpose of the performance tests was to demonstrate the noise behaviour of the interferometer. The test has been performed in vacuum. One stretch of $9000 \mathrm{~s}$ length was selected that showed little external disturbances.

Figure 4 shows the time series of the $x_{1}-x_{2}$ measurement. It is dominated by a linear thermal drift. If this drift is subtracted (right picture), it is still dominated by a slow drifting motion. These drifts are propably due to thermal effects in the metal mirror mounts. The interferometer noise is barely visible as the finite thickness of the line in the right-hand graph.
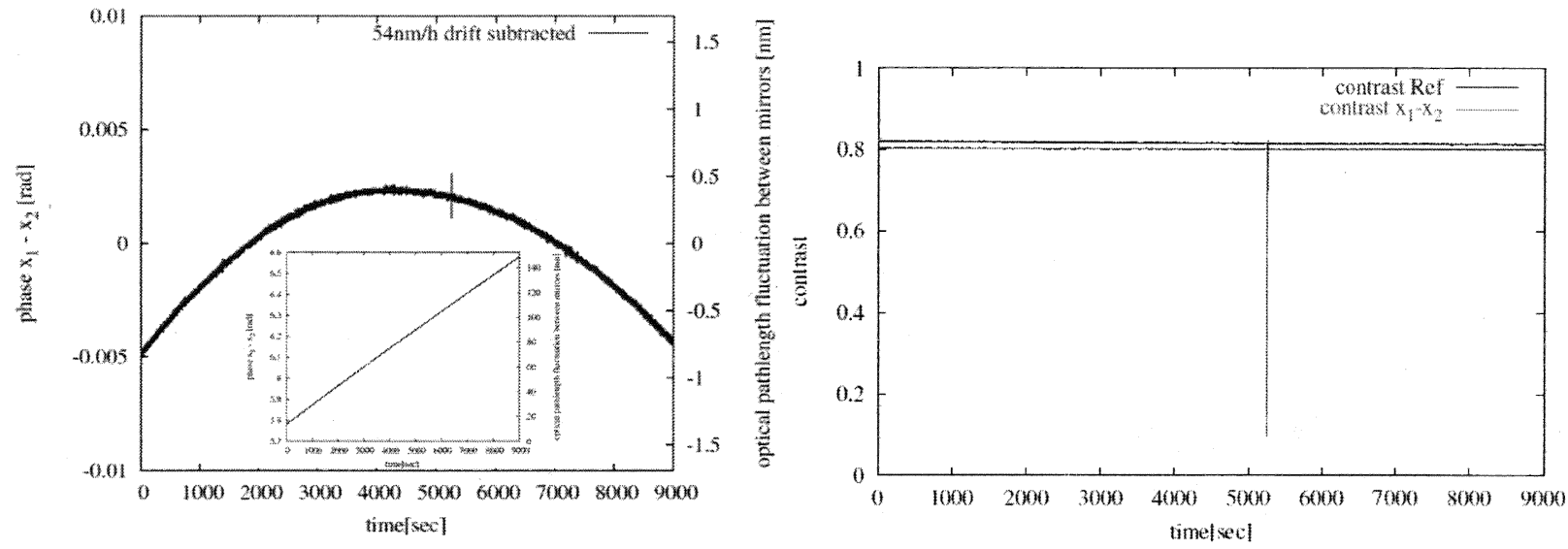

Figure 4. Left: Time series of the $x_{1}-x_{2}$ measurement: best-fit linear function (small picture) was subtracted. Right: Contrast of the $x_{1}-x_{2}$ measurement.

Figure 4 right shows the contrast of the Reference and $x_{1}-x_{2}$ interferometers. It shows a drop at about $5200 \mathrm{~s}$, which could be traced to a momentary loss of laser power (maybe due to a dirt particle in the beam path). It is remarkable how the $x_{1}-x_{2}$ measurement (Figure 4) is nearly unaffected by this interruption. This is in strong contrast to most other types of interferometer (Fabry-Perot, Michelson on dark fringe etc.) which probably would have 'lost lock' in such a situation and would have required a time-consuming and risky re-initialization.

Figure 5 shows a linear spectral density of the $x_{1}-x_{2}$ measurement. The dark solid curve shows the measurement described here. For comparison, several other curves are also shown (from top to bottom at $\left.2 \cdot 10^{-2} \mathrm{~Hz}\right)$ :

- A curve measured at TNO in a comparable configuration, but without active fiber optical pathlength (OPD) stabilization.

- The LPF mission goal.

- the previous 'best curve' with quadrant diodes, from the Glasgow prototype interferometer (also developed during the preinvestigations), measured in collaboration between Glasgow and AEI staff at Glasgow using both Glasgow and AEI equipment fro comparison.

- The LPF interferometer goal.

- The result discussed here, which represents the new 'best curve' with quadrant diodes. 
- the 'best curve', from the Glasgow prototype interferometer (also developed during this study on lab level), measured in collaboration between Glasgow and AEI at Glasgow using both Glasgow and AEI equipment. This curve was measured with single-element diodes, which consistently show a better noise behaviour than quadrant diodes, the reason for which is under investigation.

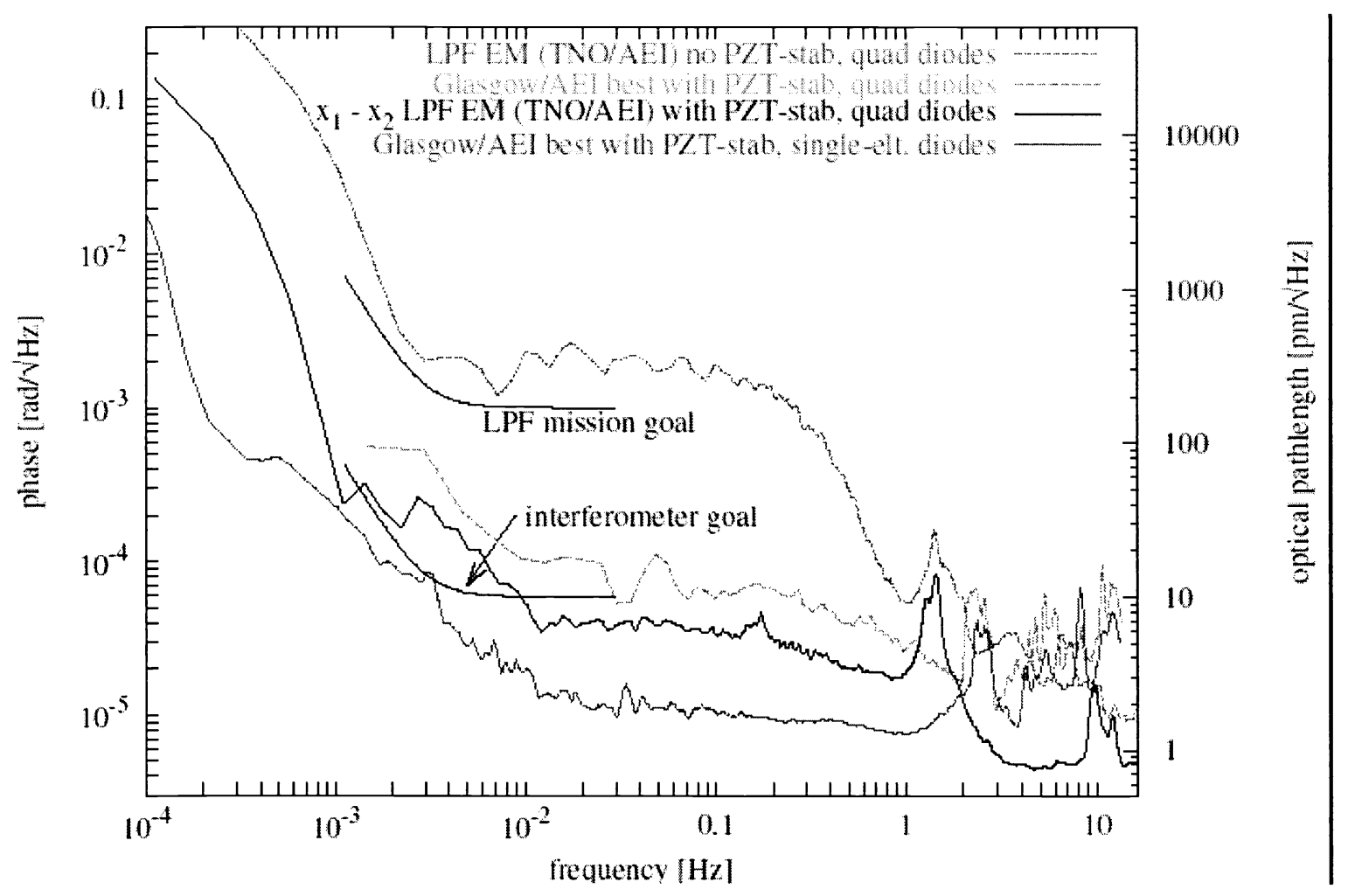

Figure 5. Linear spectral density of the $x_{1}-x_{2}$ measurement. The requirements to succeed the LPF mission are fulfilled.

Figure 6 shows a linear spectral density of the DWS alignment measurements. While we had always predicted a noise level of $10 \mathrm{nrad} / \sqrt{\mathrm{Hz}}$ (referred to the test mass misaligment), these measurements show that the noise level can be even lower than that. For comparison, $10 \mathrm{nrad}$ corresponds to an angle of $1 \mathrm{~mm}$ on $100 \mathrm{~km}$. The higher noise of the PDR alignment can be traced to the fact that this diode shows a large static DWS misalignment (due to the imperfect beam injector alignment), whereas the static DWS misalignment could be made negligible for the $x_{1}-x_{2}$ measurement using the mirror mounts.

\section{ENVIRONMENTAL PERFORMANCE VERIFICATION}

The scope of the environmental tests has been to proof that the optical bench can survive the temperature variations and the vibration levels that are going to be present during the ground, launch and orbit operations of the LISA Pathfinder mission. To this end, the test campaign consists of two separate tests: a thermo-vacuum test and a vibration test.

The following test levels have been performed: Temperature variations in the range $0-40{ }^{\circ} \mathrm{C}$, Quasi-static accelerations up to $25 \mathrm{~g}$, as well as sine and random vibration levels as shown in the table below.

Visual inspections, functional optical verifications and relative alignment check of the laser beams within the LTP interferometers at the end of the tests confirmed that the every element completely withstood the test campaign. 


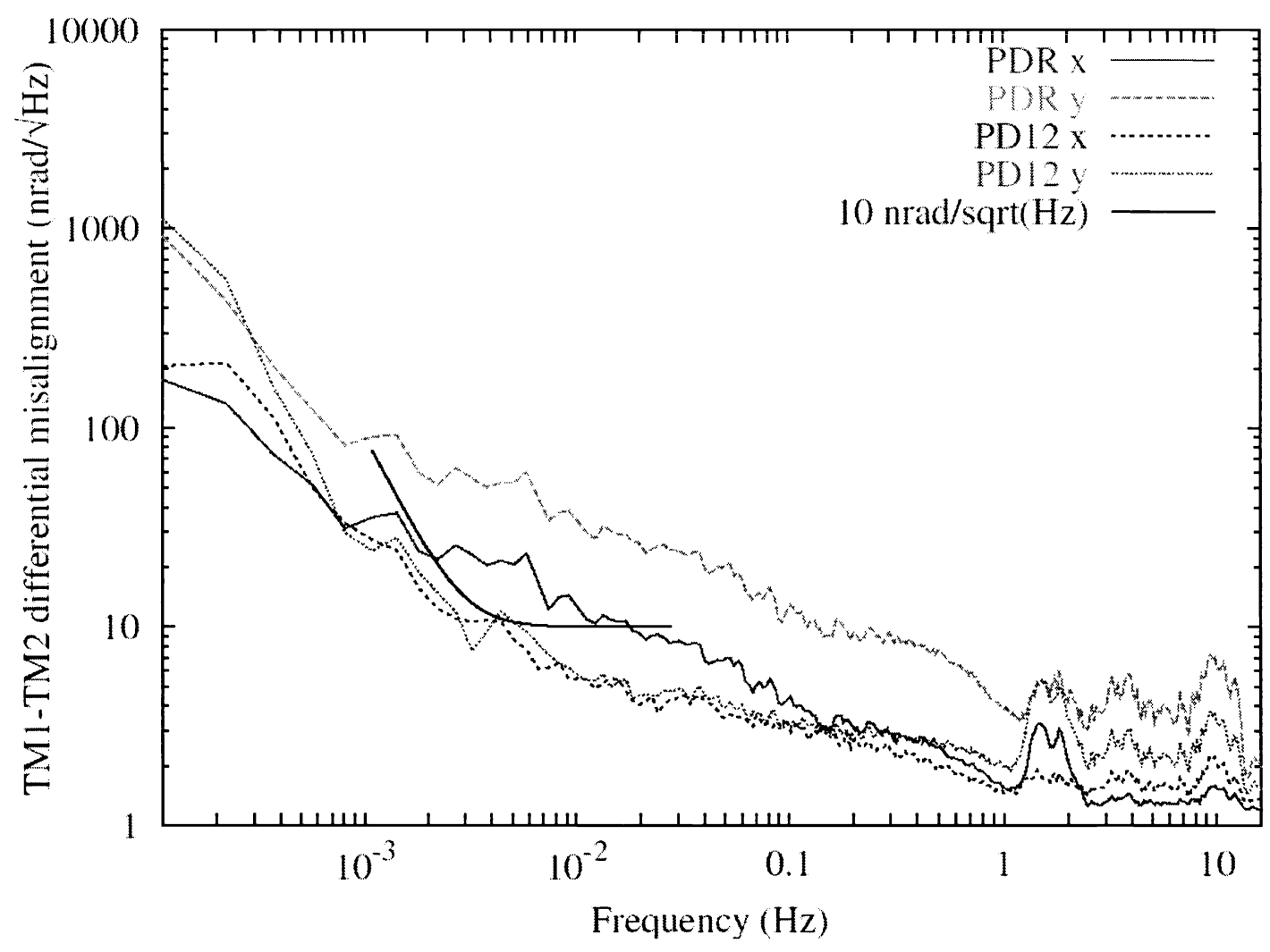

Figure 6. Linear spectral density of the DWS alignment measurements. Also this measurement verifies the requirements to succeed the LPF mission.
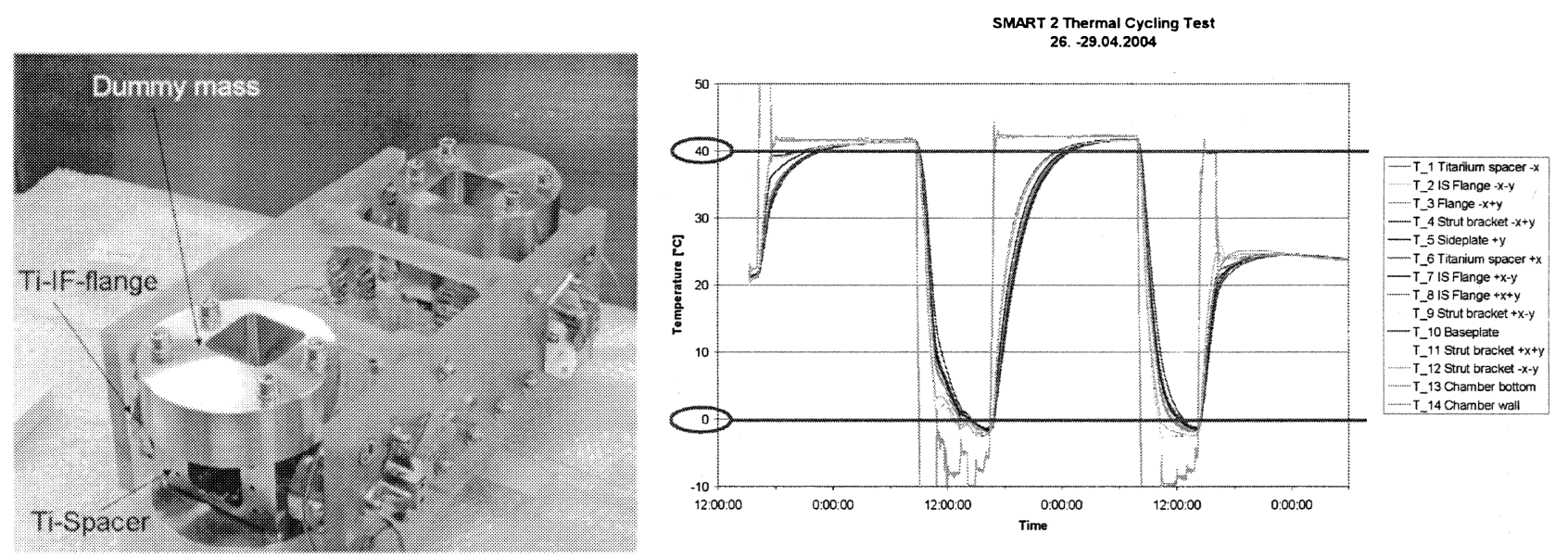

Figure 7. Left: EM OB with dummy masses. Right: Temperature readings during the TV-Test. The upper and lower limits have been reached by all sensors. 


\begin{tabular}{|c|c|c|c|}
\hline Main Tests & Freq. Range [Hz] & Input level & Duration $/$ Sweep rate \\
\hline Full level sine & $5-15$ & $\pm 11 \mathrm{~mm}$ & \\
& $15-50$ & $8 \mathrm{~g}$ & 2 oct./min., 1 sweep up \\
& $50-80$ & $6 \mathrm{~g}$ & \\
& $80-100$ & $3.5 \mathrm{~g}$ & $60 \mathrm{sec}$. \\
& $20-100$ & $+3 \mathrm{~dB} / \mathrm{oct}$ & $(8.3 \mathrm{grms})$ \\
\hline
\end{tabular}

Figure 8. Vibration levels applied to the EM OB.
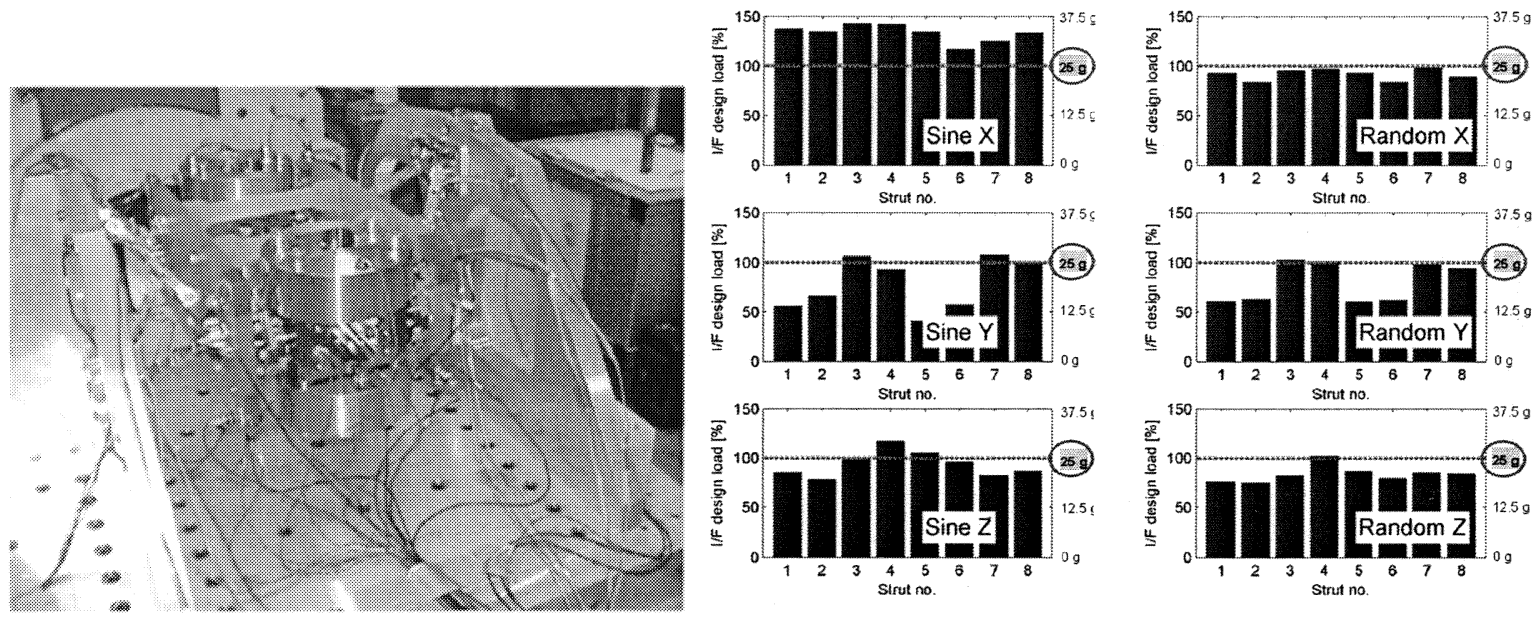

Figure 9. Left: EM OB attached to vibration table by mechanical representative struts (with respect to current FM baseline) and adapter. Cables of the accelerometers can also be seen. Right: Loads at the eight interface points during the vibration test.

Thermo-Vacuum Test (TV-Test) The TV-Test consists of a thermal cycling test of the optical bench under vacuum conditions.

This test particularly stresses the area at the titanium insert which connect the several components of the optical bench: in such a region, three different materials (Zerodur, Titanium and adhesive) shall not fail despite the largely different coefficients of thermal expansion.

Figure 7 (right) shows the temperature readings at various locations on the optical bench during the TVTest. The whole structure was able to withstand the specified temperature variation: no adhesive delamination of other type of failure was noted at the end of the test.

Vibration Test During this test the optical bench is equipped with dummy masses that are representative of the inertial sensors in terms of mass properties and stiffness ${ }^{\dagger}$.

As for the TV-Test, the most stressed regions of the structure are the locations around the titanium inserts, which represent the locations where the mechanical loads flow into the structure.

The test campaign included both sine and random vibrations along three axes. Figure 9 shows the maximum interface loads seen by each support strut during the full-level runs. The optical bench passed the test against design limit of $25 \mathrm{~g}$ in every test run. The first measured structural Eigenfrequency was at $140 \mathrm{~Hz}$.

\footnotetext{
${ }^{\dagger}$ It is worth noting that the inertial sensors (and hence the mass dummies) represent the heaviest part of the mission payload (more than 50\%). In order to provide the required opto-mechanical stability such masses must be supported by the Zerodur optical bench only.
} 


\section{CONCLUSION \& OUTLOOK}

Conclusion: The LISA pathfinder OB engineering model development and its verification was described. This tool provides to our knowledge the highest accuracy in differential displacement metrology ever achieved. The LTP EM OB structure, integration technology, optical bonding technique and laser interferometry for the relative displacement and tilt metrology between two test masses with noise levels of $10 \mathrm{pm} / \sqrt{\mathrm{Hz}}$ and $10 \mathrm{nrad} / \sqrt{\mathrm{Hz}}$ at required measurement bandwidth was verified. Environmental tests have been performed: $25 \mathrm{~g}$ loads, random and sine vibration as well as $80 \cdots 40^{\circ} \mathrm{C}$ thermal cycles. The technology readiness of the OB for the LPF flight model development is thus proven.

Outlook: The LISA pathfinder OB engineering model has to be adapted to other still ongoing LPF EM developments such as the EM Inertial Sensor. Goal is to develop the LTP EM hardware for LPF, especially a thermal shield to provide the challenging temperature stability and the attachment struts to the SC. This

is covered by an ESA contract under industrial LTP architecture lead of EADS Astrium GmbH and as prime University of Trento.

The LTP FM development already started and the remaining EM developments besides lessons learned during this study have to be implemented in the FM development plan. The planned launch for LPF is 2007.

\section{ACKNOWLEDGMENTS}

The whole EM OB team thanks S Vitale, R Dolesi and D Bortoluzzi from University of Trento for support \& fruitful discussions.

\section{REFERENCES}

1. Astrium et al: "LISA: Study of the Laser Interferometer Space Antenna, Final Technical Report", ESTEC Contract No. 13631/99/NL/MS, Report No. LI-RP-DS-009, April 2000.

2. Spero R et al.: Class. Quantum Grav. 21 (2004) S589.

3. Heinzel G et al: "Interferometry for the LISA technology package (LTP) aboard SMART-2", Class. Quantum Grav. 20 (2003) S153-S161.

4. Heinzel G et al: "The LTP interferometer and phasemeter", Class. Quantum Grav. 21 (2004) S581-S587. 